additional children often takes on new meaning, as does the decision to undergo prenatal testing. The purpose of this presentation is to explore reproductive decision-making in families who already have one child with Down syndrome (DS). Five stories from an ongoing cross-cultural study about adaptation and resilience in families of children with DS will be presented. The stories to be presented were purposefully selected because they vividly illustrate the complexity of reproductive decision-making in these families. Moreover, they show that for many families, the prenatal testing experience can be a stressful experience filled with ethical issues. Currently, there is an urgent need for more dialogue between families of children with DS and their health care providers concerning reproductive decision-making.

\title{
OC44 - Supporting the behavioural health of children: temperament-based interventions to support development and reduce risk
}

Pamela Galehouse (US) ${ }^{1}$

\section{${ }^{1}$ Seton Hall University}

Theme: School health.

Keywords: Assessment, environmental modifications, temperament.

Temperament has been described as a composite of innate characteristics best reflected in an individual's immediate response to change in his or her environment. Responses are automatic and not effected by context, ability or motivation. Some profiles of temperament present risk to developing self-control, both behavioral and emotional.

Today, nurses encounter large groups of children exposed to chaotic environments that threaten their immediate health, as well as their ability to transition into new environments and to integrate into the school and community environment. Understanding a child's unique temperament provides a framework for understanding his or her behavior and responding in a way that promotes self-understanding and behavior modification. Several successful school-based intervention programs will be identified.

In this presentation an assessment of child temperament will be reviewed, two 'challenging' temperament profiles that pose increased risk will be identified and strategies for responding to behavior in the school will be described.

\section{OC45 - Towards family-centred care in neonatal intensive care unit}

Maria Do Céu Barbieri-Figueiredol; Madalena Ramos (Portugal)2;

Branca Oliveira (Portugal) 2; Eugénia Fernandes (Portugal)2;

Florbela Neto (Portugal) ${ }^{2}$

${ }^{1}$ Escola Superior de Enfermagem do Porto; ${ }^{2}$ Centro Hospitalar de S. João, Hospital Pediátrico Integrado

Theme: Complex health care and chronic disease management. 
Keywords: Family-centred care; Neonatal Intensive Care Unit, neonatal nursing.

Family-centred care is a philosophy of care characterized by partnership and respect between healthcare professionals and families (and patients), demanding a systematic assessment of the complexity of each family, including their beliefs and values, communication style, and ability to make decisions.

This presentation will unfold the evolution of nursing care delivered at a Neonatal Intensive Care Unit of a University Hospital in the north of Portugal, since its origin in 1983 to the present day.

Some milestones will be analysed such as the inclusion of parents in the care delivery, the implementation of kangaroo care, the systematic discharge protocol or the recent award of Newborn Individualized Developmental Care and Assessment Program (NIDCAP) Certified Centre.

Next developments, starting this year, include the development of the primary nursing model for nursing staff allocation and quality audits for assessing quality of care and patient safety.

\title{
OC46 - NIDCAP at hospital São João - the journey
}

\author{
Madalena Ramos (Portugal)'; Carla Castro (Portugal)'; \\ Florbela Neto (Portugal) $)^{1}$; Ligia Silva (Portugal) \\ Fátima Clemente (Portugal) ${ }^{11}$; Hercília Guimarães (Portugal) ${ }^{1}$
}

${ }^{1}$ Centro Hospitalar de São João, Hospital Integrado de Pediatria

Theme: Complex health care and chronic disease management.

Keywords: Newborn Individualized Developmental Care and Assessment Program (NIDCAP), Neonatal Intensive Care Unit (NICU), premature.

In 2003, a group of professionals from Oporto went to Boston in the United States in order to get to know the NIDCAP program and brought it to Portugal, because they believed that individualized care is the adequate response to the developmental needs of preterm babies and their families, a concern of the unit since its inception.

The healthcare professionals were certified in the program, which began at the unit on 11 June 2011. Since then a better support system was provided to both families and professionals through systematic observations that provide guidelines for care, based on interpretation of the signs that preterm babies show.

On 1 April 2015 the São João NIDCAP Training Center opened, the only

Portuguese-speaking center in the Federation, providing training to healthcare professionals working in NICUs.

We intend to present this journey and the benefits it brought to the Hospital São João's $\mathrm{NICU}$. 